IZA DP No. 7817

Do Business Cycles Have Long-Term Impact for Particular Cohorts?

Torben M. Andersen

Jonas Maibom

Michael Svarer

Allan Sørensen

December 2013 


\title{
Do Business Cycles Have Long-Term Impact for Particular Cohorts?
}

\author{
Torben M. Andersen \\ Aarhus University and IZA \\ Jonas Maibom \\ Aarhus University \\ Michael Svarer \\ Aarhus University and IZA
}

Allan Sørensen

Aarhus University

\section{Discussion Paper No. 7817 \\ December 2013}

\author{
IZA \\ P.O. Box 7240 \\ 53072 Bonn \\ Germany \\ Phone: +49-228-3894-0 \\ Fax: +49-228-3894-180 \\ E-mail: iza@iza.org
}

\begin{abstract}
Any opinions expressed here are those of the author(s) and not those of IZA. Research published in this series may include views on policy, but the institute itself takes no institutional policy positions. The IZA research network is committed to the IZA Guiding Principles of Research Integrity.

The Institute for the Study of Labor (IZA) in Bonn is a local and virtual international research center and a place of communication between science, politics and business. IZA is an independent nonprofit organization supported by Deutsche Post Foundation. The center is associated with the University of Bonn and offers a stimulating research environment through its international network, workshops and conferences, data service, project support, research visits and doctoral program. IZA engages in (i) original and internationally competitive research in all fields of labor economics, (ii) development of policy concepts, and (iii) dissemination of research results and concepts to the interested public.
\end{abstract}

IZA Discussion Papers often represent preliminary work and are circulated to encourage discussion. Citation of such a paper should account for its provisional character. A revised version may be available directly from the author. 
IZA Discussion Paper No. 7817

December 2013

\section{ABSTRACT}

\section{Do Business Cycles Have Long-Term Impact for Particular Cohorts?*}

Will the current employment crisis produce lost generations with permanently lower labour market attachment? Taking an explicit cohort perspective and based on Danish data we do not find strong persistence in employment rates at the cohort level. Younger workers tend to be more exposed to business cycle fluctuations than older workers, but importantly they recover more quickly from such set-backs than older workers for whom persistence is stronger. Moreover, no cohorts have been disproportionately affected by exposure to a sequence of adverse shocks. An explicit account of overlapping cohorts is shown to affect assessments of persistence in aggregate employment rates.

JEL Classification: J6, E32

Keywords: persistence, lost generations, employment

Corresponding author:

Jonas Maibom

Department of Economics and Business

Aarhus University

Fuglesangs Allé 4, 2621, 5a

8210, Aarhus V

Denmark

E-mail: maibom@econ.au.dk

\footnotetext{
* We gratefully acknowledge comments and suggestions from Mette Verner and participants at CAFE, ESPE, EEA and the Danish Econometric Society meetings 2013.
} 


\section{Introduction}

The current crisis has caused sharp employment declines in many countries. A particular concern, spurred by the very high youth unemployment rates, is that the current young generation may become a lost generation in the sense that they never fully recover from the present crisis. Such a scenario entails wide social and distributional consequences, and the medium and long run implications of the current slump are thus intensively debated among unions, politicians and economists.

It is well known that past crises have produced persistent decreases in aggregate employment. Notably, the crises in the 1970s and 1980s were associated with strong persistence in the labour market, which in turn resulted in a voluminous theoretical and empirical literature on labour market persistence. Given the severeness of the current job-crisis it may be feared that persistence may be even stronger than in the past.

By persistence is understood that a change in the labour market position has longlasting effects on future labour market positions. The notion of lost generations entails that this effect pertains to specific cohorts. A particularly strong form of persistence would arise if youth entering the labour market in a slump (boom) as a result would experience a worse (better) labour market position in their remaining labour market career.

In addressing the question of persistence various interpretations or distinctions are possible. A key issue is the distinction between exogenous versus endogenous persistence. ${ }^{1}$ Exogenous persistence arises if the aggregate impulse to employment itself displays persistency, while endogenous persistence relates to whether the labour market response tends to produce persistence. In the latter case even a temporary exogenous impulse has a persistent effect on employment rates. This suggests two interpretations of the notion lost generations. One is where endogenous mechanisms in the labour market produce persistence in the labour market position. Another is where some cohorts have been exposed to a sequence of adverse (or good) shocks therefore ending up in worse (or better) positions than other cohorts. ${ }^{2}$ We consider both notions of a lost generation.

The theoretical challenge is to explain endogenous persistence; that is, why a tempo-

\footnotetext{
${ }^{1}$ In the business cycle literature there is a long-standing debate on the role of endogenous and exogenous sources of persistence. Output measures generally display strong persistence, and the standard real business cycle model can only replicate this by assuming shocks with strong persistence, see e.g. Cogley and Nason (2005). A literature has explored the source of endogenous persistence such that even temporary exogenous impulses cause a persistent response, see e.g. Andersen (2004).

${ }^{2}$ Note that the effects from booms and recessions are treated (modelled) symmetrically in the following.
} 
rary exogenous business cycle shock translates into a persistent effect on labour market variables. The literature has focused on human capital, wage formation (insider-outsider models), capital accumulation and labour market policies/institutions (for a survey of the earlier literature see Roed (1997)). Human capital depreciation during non-employment either due to depreciation of acquired skills and of firm-specific knowledge (see e.g. Pissarides (1992) and Lockwood (1991)) or due to social problems caused by the failure to be self-supporting may be an important source of cohort specific persistence and thus the possibility of lost generations. If a particular cohort is exposed to a large adverse employment shock, it follows that this cohort may find it difficult to return to employment when the business cycle normalizes, and thus it may experience a persistent lower employment rate (see also Oreopoulos, Wachter and Heisz (2012)). Our empirical model is explicitly setup so as to allow an assessment of the strength of endogenous persistence in the labour market.

The empirical literature on labour market persistence has both a macro and a micro strand. The macro literature is mainly focused on establishing whether aggregate measures, like employment and unemployment rates, display persistence. A strong form of persistence arises if, e.g., the coefficient in an $\mathrm{AR}(1)$ specification of the aggregate unemployment rate is close to unity. ${ }^{3}$ Related is the literature considering institutional and structural factors and the possibility that they account for labour market persistence (for a survey see Blanchard (2006)).

While the macro literature has yielded important insights, especially of a comparative nature on speeds of adjustment in the labour market, it suffers from the problem that it is incapable of clarifying whether the burden of persistence rests on particular cohorts. Importantly, this issue cannot be addressed by considering, for instance, the time-series properties of the (un)employment rate of a given age group (see e.g. Bernal-Verdugo et al. (2012) on youth unemployment). There may be a very strong time dependence in the unemployment rate for a particular age group, say 20-25 years, due to institutional factors. Clearly this is not necessarily implying that there is strong cohort specific persistence. The reason being that cohorts age with time, and hence to assess cohort specific persistence one has to analyse cohorts over time, and not age groups over time. ${ }^{4}$

\footnotetext{
${ }^{3}$ See e.g. Duval et al. (2006) and Guichard and Rusticelli (2010).

${ }^{4}$ This problem also appears in cross-country comparisons. Giuliano and Wachter (2012) show that although youth unemployment is much higher in France than in Germany, the subsequent labour market performance for older age groups is quite similar in the two countries.
} 
The micro literature exploits individual data to identify employment prospects and possible persistence or path-dependencies depending on individual characteristics. In the seminal paper by Heckman and Borjas (1980) a central question is whether unemployment increases the risk of being unemployed in the future. Recently, analyses of the consequences from entering the labour market in a recession have re-flourished (see e.g. Genda, Kondo and Otha (2008), Oreopoulos, Wachter and Heisz (2012), Bell and Blanchflower (2011) and Liu, Salvanes and Sørensen (2013)). The general finding is both a shortand a long-term individual level effect on employment and earnings. The effects differ across educational groups, and especially workers with intermediate levels of education are in an exposed position. This part of the literature is therefore closely connected with the idea of lost generations. As the youth have less experience and are potentially less efficient job seekers, cohorts graduating in a recession may be in a particularly exposed position. The micro approach suffers from two shortcomings. First, persistence at the individual level does not have clear-cut implications for possible persistence at the cohort level. This point has been raised several times in the literature, recently Blundell and Stoker (2007) point out that the aggregation link between individual behaviour, heterogeneity in many dimensions and aggregate dynamics is certainly not trivial. Second, it does not allow a separation between endogenous and exogenous sources of persistence.

To overcome the problems associated with both the micro and the macro approach, we propose an intermediary approach - the meso level - by considering employment responses to the business cycles and dynamics at the cohort level. We take explicit outset in the life-cycle pattern of employment (inverted U-shaped relation between employment rates and age) and consider whether the labour market situation for a cohort at a particular age can affect the same cohort's labour market position at a later age, and possibly through their remaining labour market history. By controlling for the driver of the business cycle shocks (exogenous) as well as endogenous mechanisms in the labour market, we are able to consider both notions of lost generations discussed above.

The meso level of aggregation has advantages relative to both the macro and the micro approach. The macro approach relying on aggregate (un)employment rates suffers by construction from being silent about potential dynamics at the cohort level, although important for understanding the evolution of the aggregate (un)employment rate over time. We underline the latter point by showing that evaluating persistence from an explicit cohort perspective leads to a different assessment than implied by the aggregate time-series approach. The micro approach focussing on persistence at the individual level 
does not capture the processes applying at the level of cohorts. The individual path may depend on idiosyncratic individual shocks, whereas we are interested in the response to aggregate labour market shocks and to separate endogenous from exogenous sources of persistence.

Analyses based on individual data are critically dependent on having the proper control variables capturing individual characteristics and labour market histories, whereas an analysis at the meso or cohort specific level is less vulnerable to these data requirements under the assumption that the distribution of these individual characteristics is (reasonably) constant across cohorts. ${ }^{5}$ By analysing cohort specific performance we focus on what survives aggregation from the individual level and becomes embedded in the employment record for a particular cohort. This also implies that our approach allows for peer effects (or externalities) among individuals within specific cohorts and our results will therefore capture more than individual scarring effects. Our procedure also makes it more easy to control for the typical life-cycle pattern of employment, which has a strong age-gradient not to be interpreted as persistence. For instance, younger aged workers are likely to have a lower employment level (and maybe even less stable) simply due to the fact that they have just entered the labour market. Finally knowledge of dynamics at the cohort level is important from a policy perspective, especially whether policies should be targeted towards cohorts/age groups.

We use Danish register data for the entire labour force population for the years 19802008. Our key variable is the employment rate. We choose the employment rate rather than the unemployment rate, as the latter does not take the labour force participation decision into account and is associated with various measurement problems in the time frame of our study (it is affected by institutional changes). Moreover, we distinguish by gender and educational levels to identify possible differences in exposure to shocks and in persistence across key socioeconomic groups in the labour market. In addition to the employment rate we have considered earnings at the cohort level. However, as the findings were qualitatively similar, we report only the findings for the employment rate.

Our results show that although the adjustment of employment shocks is gradual, there is no evidence of strong endogenous persistence and thus lost generations in this sense. In

\footnotetext{
${ }^{5}$ The sample is split by different age groups, and by having cohort specific covariates we allow for differences between cohorts, cf. below. Furthermore our approach allows us to use data covering almost 30 years, and thereby we use variation from several recessions and booms to asses the persistence in employment rates. This would naturally be very challenging in an individual level analysis.
} 
line with the literature, we find that younger workers tend to be more exposed to business cycle fluctuations than older workers. Importantly, however, we find that younger workers recover more quickly from such set-backs in contrast to older workers. For elderly women with low or medium levels of education, employment rates may be permanently affected by business cycle changes. These findings illustrate the very different costs business cycle fluctuations can have over the life-cycle. Finally, we also find that there is not a strong case of certain cohorts becoming lost generations as a result of having been exposed to a sequence of adverse shocks in vulnerable ages.

The paper is organized as follows: Section 2 describes the data and some facts. Section 3 sets up the econometric model, and Section 4 describes the estimation techniques applied. Section 5 contains the estimation results, and Section 6 considers the specific question of lost generations. Finally, Section 7 concludes.

Our empirical analysis is based on a Danish register-based annual matched-employeremployee panel covering 1980-2008. This panel contains the whole of the Danish labour force. The unit of observation is a given individual in a given year with measurements generally referring to the last week of November. We aggregate across individuals and compute the employment to population ratio (EPR) for each cohort in a given year. We restrict the analysis to cohorts born from 1935 to 1980 as this secures a minimum of 9 observations for each cohort. ${ }^{6}$

The sample is split according to gender and education (see Appendix A). The educational level of the individual is defined by the highest obtained education around age 30 (or later if not present). Individuals who have education similar to a High School degree or less as their highest education are classified as being low educated, whereas individuals having education similar to a bachelor degree or above are classified as being highly educated. The remaining individuals with intermediate levels of education are classified as being medium educated.

\subsection{Employment rates in a life-cycle perspective}

The basic life-cycle pattern for cohorts is illustrated in the left panel of Figure 1 for males and females, respectively.

\footnotetext{
${ }^{6}$ Note that our sample have truncated spells; i.e. young generations with incomplete spells and older generations where the previous employment spell is not known. Figure A.1 in the appendix gives a visual representation of the data.
} 
Figure 1: Employment to population Rates by AGe And year
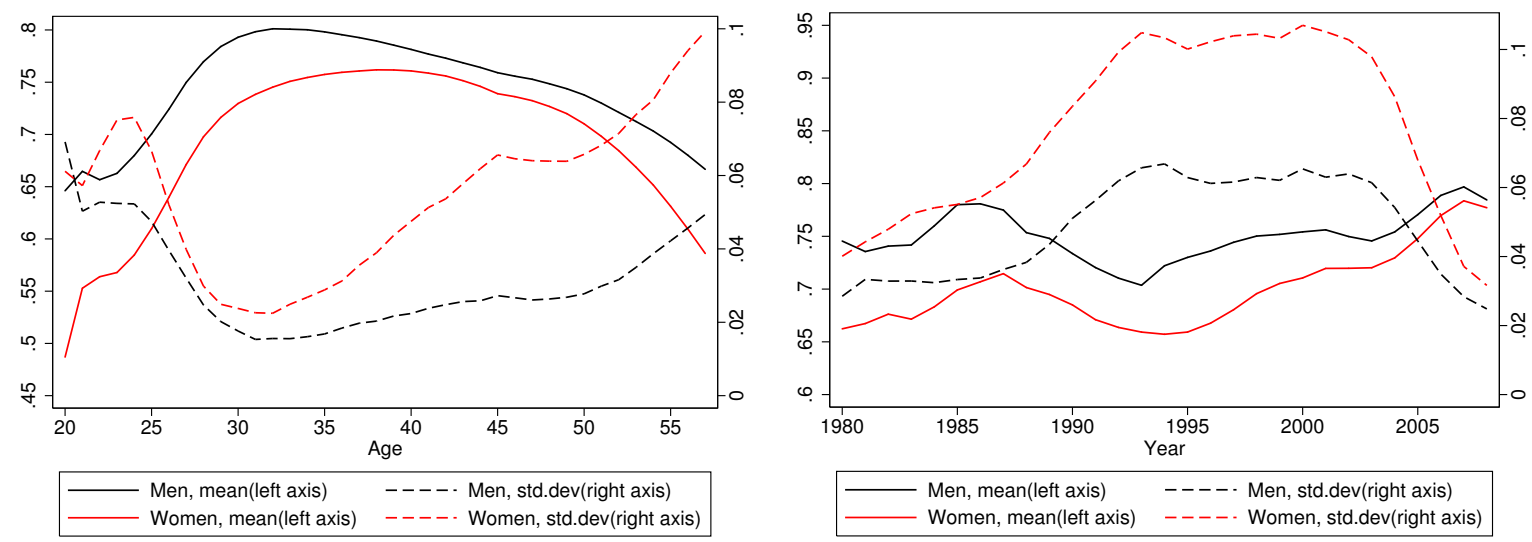

Note: For the age specific rates standard deviations are computed from variation across time for a given age group, and for the year specific rates standard deviations are computed from variations in different age groups in a given year.

The evolution of the mean EPR displays the expected inverted U-form with employment rates first increasing and later decreasing with age, reflecting entry and exit into the labour market. The mean EPR for men is higher than for women for all ages, but the inverted U-shape is very similar. The standard deviation around the mean follows a U-form attaining its lowest value for the age group 30-35, where the average employment frequency is also close to its peak. The low standard deviation for the middle-aged (prime aged) suggests that there is no strong persistence running through the entire work life for a cohort. That is, irrespective of the employment trajectory during the early years, all cohorts have approximately the same employment rate when becoming 30-35 years old (the standard deviation is around $2 \%$ ). In particular, it is noteworthy that this applies also to cohorts experiencing low employment rate for a number of years when young, cf. below. The low variability in EPR for middle-aged also shows that this age group is not significantly affected by variations in the labour market situation. Later in life the variation around the mean increases again; for men it reaches the same level as in the early years, whereas there is much more variation around the mean for women at later ages.

The right panel of Figure 1 presents the employment rates by year. The Danish economy experienced a large economic crisis through the late 80's and early 90's which caused a sharp and persistent drop in the employment rate (see Figure A.1 in the appendix for output gap during the time period). There is a tendency to convergence in employment rates between genders in the last years included in the sample. The standard deviation 
(which is now across age groups in a given year) displays a counter-cyclical tendency, suggesting that age groups are differently affected by cyclical variations.

The age dependent employment rates are shown for different educational groups in Figure 2. Both for males and females the mean employment rate is increasing in the level of education. Figure 2 also shows that the highly educated, as expected, both enter and leave the labour market later than other educational groups. Moreover, this group has a much lower variation in employment rates above the age of 30 . The larger variation before the age of 30 is likely to reflect differences in non-employment timing (e.g. education or unemployment) and length and its relation to the business cycle. Notice the U-form of the standard deviation which again reveals a convergence in cohort specific employment levels at middle ages, whereas variation between cohorts is much more pronounced in early and later ages especially for the low and medium educated.

\section{Figure 2: Employment to POPUlation RATES By EDUCATiOn AND AGE}

\section{Women}

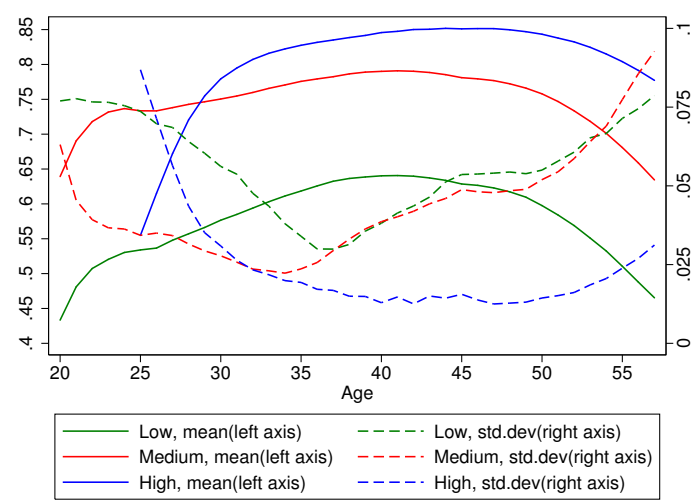

Men

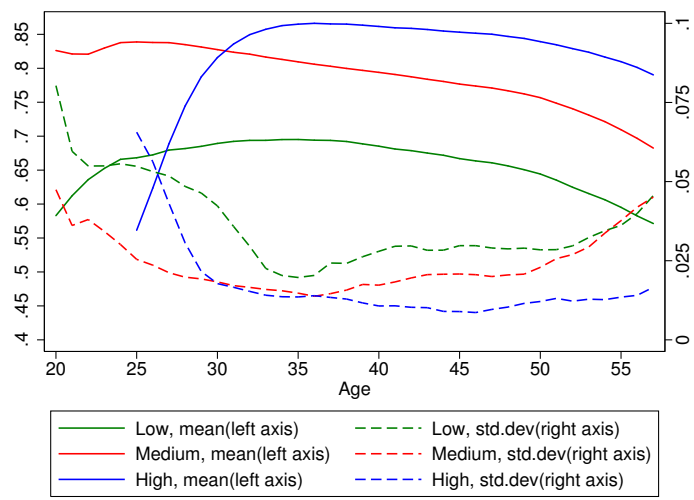

Considering the evolution of employment rates for different educational groups over time (Figure 3) it is seen that we have variation for all educational levels, although for the highly educated group this is likely to be primarily related to the young age groups (see Figure 2). The standard deviation has a weak counter-cyclical pattern for all educational groups. ${ }^{7}$

\footnotetext{
${ }^{7}$ The spike in the standard deviation for the high education group is due to the fact that our sample contains primarily younger aged workers in later years, see Figure A.2.
} 
Women

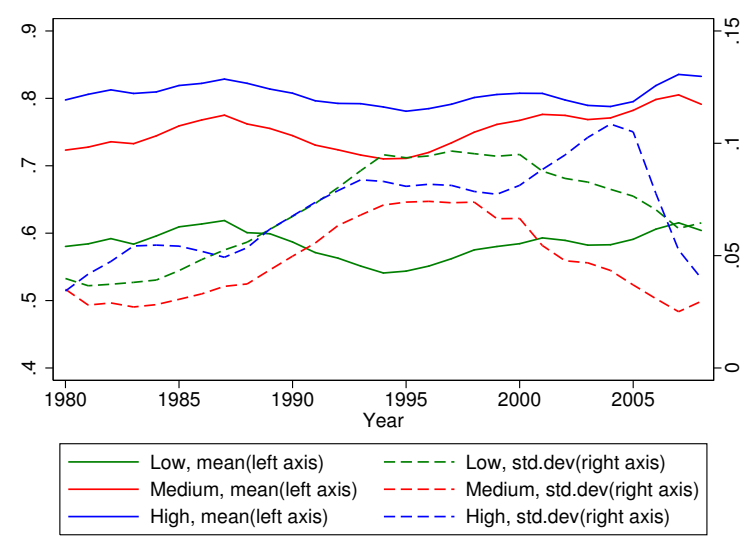

Men

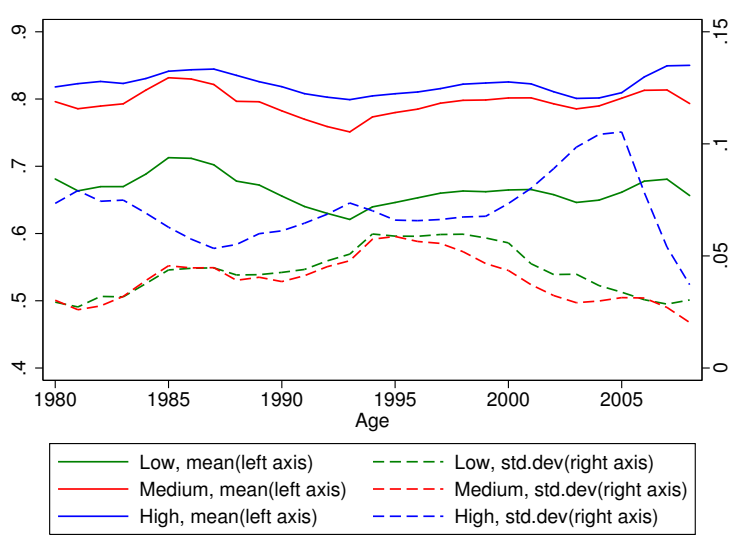

A simple and intuitive way to consider the possible path dependence in employment rates is to consider the employment trajectories for specific cohorts. Figure 4 plots the age dependent employment rates for those cohorts in the sample that experienced the highest and smallest average EPRs in their 20s. The mean reversion around the ages 3035 is easily seen from the figure; that is, both cohorts that start above and below average eventually adjust towards the mean EPRs.

\section{Figure 4: Employment to Population Rates for Selected cohorts}
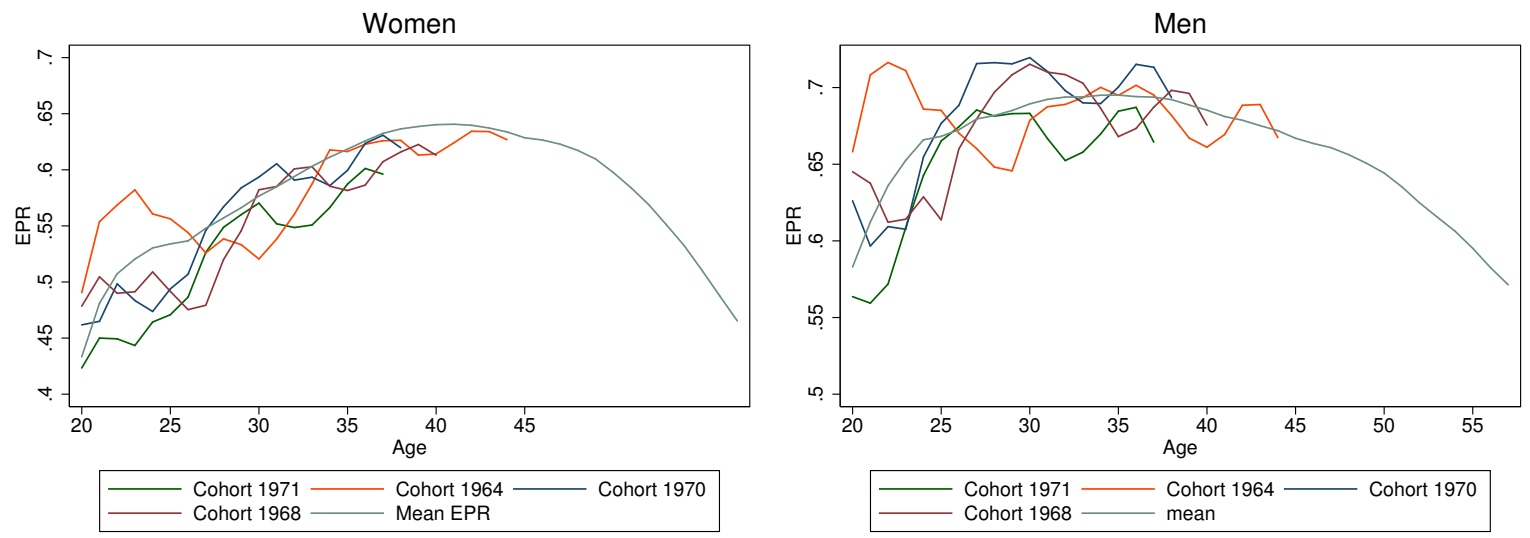

Our empirical strategy builds on the findings presented above. We explicitly model the life-cycle pattern in employment rates. Since we do not have complete life-cycle histories in our sample and to accommodate different dynamic patterns across the life-cycle, we split data into sub-samples depending on age: young and old. The young sample consists 
of cohorts with a minimum of 6 observations in the age range 20-35 (i.e. cohorts 19501980), and the old sample consists of cohorts with a minimum of 6 observations in the age range 45-57 (cohorts 1935-1958). The division of the sub-samples can also be seen as a way to explicitly distinguish between the various cohorts and the ages for which we have data. By construction our sample is, in some sense, selected as the observations we have for older ages are represented by older cohorts, and likewise for the youngest - the sample splitting makes this even clearer. To the extent that older cohorts are not representative for older workers in general beyond the intergenerational differences that we allow for in our empirical model (e.g. unobserved heterogeneity), our results would be affected by this selection. One way to phrase the central question regarding selection is whether, for instance, the 1940 generation systematically underperforms because of selection of some kind, or because they are simply the oldest available workers.

\section{Cohort specific persistence in employment}

The model we use in the empirical analysis is setup with the aim to capture the essential life-cycle pattern of employment rates (cf. Figure 1), but also to allow for business cycle impacts as well as employment dynamics. As mentioned above, we split the sample by age to account for the possible different performances of different age groups. In addition, we estimate the model separately for the three educational groups.

Our strategy is to estimate a generic relation between the employment ratio and age that takes the variation in the time and age domain presented in Figure 1 into account. The EPR, $e_{s, i, t}$, for a given gender $s$, cohort $i$ (birth year) at a given point in time $t$ depends on both the business cycle situation measured by the output gap $\left(y_{t}\right)$ and the stage in the life-cycle given by the age $a_{i, t}$. The underlying labour market structure and policies are embedded in the relationship, but various specific policy measures may apply for certain age groups in part of the sample period $\left(z_{i, t}\right)$, cf. below.

$$
\begin{aligned}
e_{s, i, t} & =\delta e_{s, i, t-1}+\kappa_{0} \cdot a_{i, t}+\kappa_{1} \cdot a_{i, t}^{2}+\theta \cdot I\left(x_{2} \geq a_{i, t} \geq x_{1}\right) \cdot y_{t}+\gamma \cdot z_{i, t} \\
& +\beta_{0} \cdot y_{t}+\beta_{1} \cdot y_{t-1}+\beta_{2} \cdot y_{t} \cdot s_{i}+\beta_{3} \cdot y_{t-1} \cdot s_{i}+\psi \cdot s_{i}+\eta_{s, i}+\epsilon_{s, i, t}
\end{aligned}
$$

We allow for the output gap to interact with age $(\theta)$ and gender $\left(\beta_{2}, \beta_{3}\right)$. The interaction term is implemented as $I\left(25 \leq a_{i t} \leq 30\right) \cdot y_{t}$ and $I\left(30 \leq a_{i t} \leq 35\right) \cdot y_{t}$ in the young 
sample and $I\left(50 \leq a_{i t} \leq 54\right) \cdot y_{t}$ and $I\left(55 \leq a_{i t} \leq 57\right) \cdot y_{t}$ in the old sample. $\eta_{s, i}$ represents cohort/gender specific unobserved heterogeneity, and $\epsilon_{s, i, t}$ is the error term.

Note that dynamics of the employment rate $\left(e_{s, i, t}\right)$ driven by aggregate shocks are captured by an ARMA-process embedded $\operatorname{in}^{8}(1)\left(\delta e_{s, i, t-1}+\beta_{0} \cdot y_{t}+\beta_{1} \cdot y_{t-1}\right)$, where the latter two terms (the MA part) are the exogenous driving forces, and the first term (the AR part) captures the endogenous adjustment mechanism. For $\delta$ close to zero the dynamics of employment rates (around the generic age relationship) are mainly driven by the exogenous impulse, while for $\delta$ close to one even temporary exogenous influences may have long lasting effects on the employment rates of a particular cohort (endogenous persistence) ${ }^{9}$

To represent the state of the economy $\left(y_{t}\right)$ we use the measure of the output gap used by the Danish Economic Council. The output gap represents the deviation of the economy from its current "steady state". This also implies that this measure will, to some extent, control for structural changes in the economy in our time frame. We have tried several other measures for the economic activity, including the OECD output gap measure and gaps generated by use of HP-filters. We have not found measures that will alter our results below noticeably. We include timedummies for some specific years and a time trend in our final specification to allow for unmodelled time effects in the employment rates. This will be further discussed later. ${ }^{10}$ Notice that there are potential simultaneity problems in using the output gap as an explanatory variable for the employment level, therefore we instrument it with lagged levels of the output gap, see below. The business cycle measure is only included with one lag in the reported estimation as we have found that this was sufficient to capture the dynamic process. We will comment further on this restriction below.

We have considered various specific policy programmes ${ }^{11}$ which have been targeted towards specific age groups during the sample period. A so-called transition scheme for elderly workers was found to be effective at the cohort level. This scheme was in effect in the period 1992-1995 for unemployed individuals in the age group 50-60 years. This

\footnotetext{
${ }^{8}$ Neglecting the cross-terms to simplify.

${ }^{9}$ Naturally this depends on $\beta_{1}$. This illustrates that it is important to allow for both adjustment mechanisms. For instance, excluding $\beta_{1}$ would have important implications for the estimated size of both the endogenous and exogenous effects.

${ }^{10}$ Naturally there is an identification problem for the output gap if one employs time dummies for the whole of the time period

${ }^{11}$ We also considered a youth package introduced in 1996, but did not find it to have significant effects.
} 
scheme allowed the eligible to extend the unemployment benefit period bridging to the statutory early retirement age (60 years). The transition scheme for elderly is found to have a significant negative effect on the EPR (and further analysis shows that this applies mainly for females). This is included in the results reported for the old sample below.

The panel structure of our data allows us to differentiate between "true" dynamics and factors that vary across, but not within, cohorts over time even though such factors are unobservable. Therefore $\eta_{s, i}$ represents life-long differences between cohorts. One way to interpret this coefficient is that it indicates structural differences between cohorts (i.e. a difference in the quality of their education, gender roles, difference in initial conditions etc.). Leaving such effects unmodelled will imply an over-prediction of persistence as the systematic constant part of the error term will be subscribed to dependence on past values of $e_{s, i, t-1}$ (this way standard OLS will give us an upper bound on the persistence coefficient). The central econometric problem above is therefore to distinguish between unobserved heterogeneity and state-dependency captured in $\eta_{s, i}$ and $\delta$, respectively. We will present our identifying assumptions in the next section.

Finally, note that we do not use time dummies for the whole of the period 1981-2008 due to the fact that we also wish to identify the effect of the output gap on the EPRs and the interaction with gender. Therefore we only employ time dummies for specific years where diagnostic plots of our residuals suggest that the measures related to output do not control sufficiently well for the aggregate time effects. To the extent that the time trend, output gap and time dummies do not control sufficiently well for time effects between individuals our results might be biased. ${ }^{12}$

\section{Estimation}

This section gives a brief introduction to the ideas behind the difference and system $G M M$ estimators with particular focus on the identifying assumptions. The estimators are designed for samples where the cross-section dimension is large, therefore we also discuss the dangers associated with the use of these estimators in a limited sample. Finally we discuss how the preferred specifications were chosen in the estimation process.

The model presented in equation (1) suffers from a general identification problem due

\footnotetext{
${ }^{12}$ We have tried a sequential approach where we use a full set of time dummies to extract all variation over time and subsequently regress the output gap on these estimates; this procedure results in very similar estimates.
} 
to the unobserved cohort specific effect $\left(\eta_{s, i}\right)$, which by construction is correlated with the lagged dependent variable and potentially also other explanatory variables. Standard panel procedures (FE estimators estimated using within transformations) have been widely noted to be downward biased (see e.g. Hayasi (2000)) due to the correlation between the demeaned lagged dependent variable and the transformed error term. This correlation arises as any persistent deviation from the "true" cohort mean will be mischaracterized as a part of the estimated fixed effect in finite samples thus reducing the estimate of $\delta$. The FE estimates and standard OLS estimates of (1) are still useful in the sense that they specify a range in which the parameter estimates should lie if the model is correctly specified.

\subsection{Difference and System GMM}

To address the identification problem mentioned above, one estimation procedure transforms the empirical model into first differences and then uses various instrumental strategies to identify $\delta$. Consider a simple $\operatorname{AR}(1)$ process with unobserved heterogeneity in first differences (we exclude the gender dimension and explanatory variables in the following to economize on space, but results easily generalize):

$$
\begin{aligned}
e_{i, t}-e_{i, t-1} & =\delta\left(e_{i, t-1}-e_{i, t-2}\right)+\left(\eta_{i}-\eta_{i}\right)+\left(\epsilon_{i, t}-\epsilon_{i, t-1}\right) \\
E\left(\eta_{i}\right) & =E\left(\epsilon_{i t}\right)=E\left(\eta_{i} \epsilon_{i t}\right)=0
\end{aligned}
$$

Note that equation (2) still produces biased estimates using least squares routines due to the correlation between $\epsilon_{i, t-1}$ and $e_{i, t-1} . \quad \delta$ can, however, be consistently estimated using a GMM/IV procedure based on the assumption that

$$
E\left(e_{i \tau} \triangle \epsilon_{i t}\right)=0 \text { for } \tau \leq t-2
$$

This procedure follows the work of Anderson \& Hsiao (1981) and Arrelano \& Bond (1991) and is often referred to as difference GMM. The crucial assumption is that the error term is truly idiosyncratic and independent across cohorts and time. It implies that we from $t=3$ and onwards will have an increasing number of instruments for each subsequent period (where $t=1$ measures the first observation we have for a given cohort). In period 3 we have $e_{i 1}$ as the only instrument for $\triangle e_{i, t-1}$, in period 4 we have $e_{i 2}$ and $e_{i 1}$, and so on. This way the number of instruments increases linearly in t. Finally one can also 
allow for serial correlation in the error term in the form of $\epsilon_{i t}=v_{i t}+\rho v_{i t-1}$ by changing the identifying moment conditions such that $E\left(e_{i \tau} \triangle \epsilon_{i t}\right)=0$ for $\tau \leq t-3$ and this way change the instrument set. Our results below are robust to both characterizations of the error term.

Blundell \& Bond (1998) show that the procedure above could cause large finite sample biases when using the levels of the dependent variable as an instrument in the differenced equation in models with moderately persistent series and moderately short panels due to a problem of weak instruments. In these cases the lagged dependent variable is simply a bad predictor for the current change in levels, and the estimates become biased in the direction of the within estimator. In line with Arrelano \& Bover (1995), Blundell \& Bond (1998) show that there are likely to be efficiency gains and bias reductions from incorporating more "informative" moment conditions.

They suggest a system GMM estimator that combines both the equation in levels and the equation in first differences. Here $\triangle e_{i \tau}$ is used as an instrument for $e_{i \tau}$ in the level equation and $e_{i \tau-1}$ is used as an instrument for $\Delta e_{i \tau}$ in the difference equation. The moment conditions related to the different equations are estimated jointly. This approach corresponds to the following moment conditions:

$$
\begin{aligned}
E\left(e_{i, \tau} \triangle \epsilon_{i, t}\right) & =0 \text { for } \tau \leq t-2 \\
E\left(\triangle e_{i t-\tau}\left(\eta_{i}+\epsilon_{i t}\right)\right) & =0 \text { for } \tau=1 \text { and } t \geq 3
\end{aligned}
$$

The additional level equation $\left(\eta_{i}+\epsilon_{i t}\right)$ implies that we get an extra moment condition for each $t$ compared to difference GMM (as later lags of the instrument become redundant when used together with the difference equation). Note that the validity of the moment conditions used in difference GMM and the extra set of moment conditions implied by system GMM can be phrased as restrictions on the initial condition for the process generating $e_{i 1}$. For difference GMM this amounts to the assumption that the initial level of $e_{i 1}$ is uncorrelated with $\epsilon_{i t} \forall t$, which is fulfilled if the error-term is truly idiosyncratic or $\mathrm{MA}(1)$. For system $G M M$ the additional requirement is that $\triangle e_{i t 1}$ is uncorrelated with $\eta_{i}$ such that the initial deviations from the long-run mean of the process are not correlated with the long-run mean itself. Arrelano \& Bover (1995) link this to a requirement of stationarity of the process of the instrument, such that we require the first moments of the instruments to be time-invariant (conditional on e.g. common year dummies). Relating the assumptions to our model in (1) they imply that differences in the changes in the EPR $(e)$ between cohorts are not related to structural differences embedded in $\eta_{i}$. 
The procedures presented above easily extend to the case with explanatory variables, which will generally be used as "instruments" for themselves. The moment conditions related to the explanatory variables depend on the assumptions made on the relationship between these and $\epsilon_{i t} \forall t$. If the regressors are treated as predetermined (such that present values of $\epsilon_{i t}$ potentially affect future values of $\left.x_{i t}^{\prime}\right)$, the conditions are $E\left(x_{i \tau} \triangle \epsilon_{i t}\right)=$ 0 for $\tau \leq t-1$ when no lags appear in $x_{i \tau}$ for difference GMM. In our preferred specification we treat explanatory variables related to the output gap as predetermined and potentially contemporaneously endogenous. Age $\left(a_{i t}\right)$, gender $\left(s_{i}\right)$ and policy programmes $\left(z_{i t}\right)$ are treated as strictly exogenous. ${ }^{13}$

On the basis of the presented moment conditions, we form a generalized metric and solve the minimization problem using the GMM framework. We do not use optimal (twostep) GMM estimators due to the limited sample size in the cross-section dimension that will generally make our estimators worse behaved. This is due to the fact that we are essentially estimating fourth moments of the underlying distribution when determining the weight matrix (see e.g. Hayasi (2000)). Our weight matrix is therefore the Identity Matrix in difference GMM and a slightly modified version in system GMM (see e.g. Arrelano \& Bover (1995)).

\subsection{Estimation Procedures}

Due to a number of over-identifying restrictions (the number of moment conditions exceeds the number of estimated parameters) in the estimators it is possible to apply standard tests to make inference on the validity of these restrictions. Generally these tests rely on large samples, and they should therefore be used with caution. Specification tests that barely exceed "conventional significance levels" can be misleading as these tests are known to be undersized (the test hardly never rejects the hypothesis) when the instrument count is high. As we have limited data in the cross-section dimension, we will also limit the number of lags of the dependent variable that we include as an instrument. The number of instruments used is generally higher than the number of cohort/gender units in the data (but naturally not observations). A large number of instruments compared to the sample is likely to over-fit endogenous variables and also weaken the Hansen test of over-identifying restrictions. We follow the practice suggested by Roodman (2009) and test each specification for the sensitivity to reductions in the number of instruments.

\footnotetext{
${ }^{13}$ We have tested the assumption on $z_{i t}$, and our results are robust to this assumption.
} 
As a standard we use a maximum of two lags of the (differenced) dependent variable as an instrument for the equation in differences (levels). Furthermore, we try various lags in order to allow for the presence of unaccounted for MA(1) errors in $\epsilon_{i t}$. Our results below are robust to these changes. The standard Sargan and difference Sargan tests are inconsistent in the case of heteroskedasticity, and therefore we also use the Hansen test as a guideline (even though they require estimations using a two-step GMM procedure which we generally do not use). Furthermore, we use the test for serial correlation developed by Arrelano \& Bond (1991) to determine the validity of our identifying assumptions regarding serial correlation in the error term. The result from these tests and various robustness exercises are reported in Appendix B.

Our preferred specification is the system GMM estimator because we find that the difference GMM estimates are much less precise and in some cases below the FE-estimates. Hayakawa (2006) shows that the system GMM estimator generally has a smaller bias in small samples (especially when $\sigma_{\eta}^{2}$ and $\sigma_{\epsilon}^{2}$ are roughly equal), and this might explain why these estimates are generally better behaved in our case.

\section{Estimation results}

This section presents our estimation results for the system GMM estimator. ${ }^{14}$ As noted above the sample is split by age and education. The robustness of the results to alternative specification of the dynamic structure of (1) is discussed in Appendix B, but generally the dynamics from these specifications are very similar to those reported below.

\subsection{Results for the young sample}

Our preferred estimates for the young sample are reported in Table 1. Note that the model pools the data for women and men $^{15}$, but allows for different responses for the two groups $\left(\beta_{1}, \beta_{2}\right)$, cf. (1).

\footnotetext{
${ }^{14}$ Extended estimation output with results from FE estimation, OLS and difference GMM are available upon request.

${ }^{15}$ We have also estimated the model separately for women and men, but the results are qualitatively similar.
} 
TABle 1: Results For the young SAMPle

\begin{tabular}{llccc}
\hline \hline & & Low educ. & Medium educ. & High educ. \\
\hline$\delta$ & Lagged EPR $\left(e_{s, i, t-1}\right)$ & $0.625^{* *}$ & $0.470^{* *}$ & $0.654^{* *}$ \\
& & $(0.0484)$ & $(0.0442)$ & $(0.0389)$ \\
$\beta_{0} \quad$ Output gap $\left(y_{t}\right)$ & $0.534^{* *}$ & $0.708^{* *}$ & $0.476^{* *}$ \\
& & $(0.0710)$ & $(0.0639)$ & $(0.0589)$ \\
$\beta_{1} \quad$ Lagged output gap $\left(y_{t-1}\right)$ & -0.0640 & 0.0665 & $-0.0939^{*}$ \\
& & $(0.0457)$ & $(0.0498)$ & $(0.0478)$ \\
$\beta_{2}$ & Output gap $\times$ male $\left(y_{t} \cdot s_{i}\right)$ & $0.526^{* *}$ & $0.354^{* *}$ & 0.100 \\
& & $(0.0778)$ & $(0.0695)$ & $(0.0669)$ \\
$\beta_{3}$ & Lagged output gap $\times$ male $\left(y_{t-1} \cdot s_{i}\right)$ & $-0.647^{* *}$ & $-0.622^{* *}$ & $-0.206^{* *}$ \\
& & $(0.0505)$ & $(0.0489)$ & $(0.0689)$ \\
$\theta$ & Age dummy $(25-30) \times$ output gap & 0.0514 & $-0.123^{*}$ & \\
& & $(0.0726)$ & $(0.0730)$ & \\
$\theta$ & Age dummy $(30-35) \times$ output gap & $-0.271^{* *}$ & $-0.326^{* *}$ & $-0.0676^{* *}$ \\
& & $(0.0769)$ & $(0.0640)$ & $(0.0331)$ \\
$\kappa_{0}$ & Age $\left(a_{i, t}\right)$ & -2.261 & -1.062 & $59.11^{* *}$ \\
& & $(3.095)$ & $(2.701)$ & $(12.48)$ \\
$\kappa_{1}$ & Age squared $\left(a_{i, t}^{2}\right)$ & $0.869^{*}$ & 0.306 & $-9.079^{* *}$ \\
& Male $\left(s_{i}\right)$ & $(0.480)$ & $(0.492)$ & $(1.855)$ \\
& Trend & $4.042^{* *}$ & $3.959^{* *}$ & $1.429^{* *}$ \\
& & $(0.629)$ & $(0.468)$ & $(0.155)$ \\
& & $-0.232^{* *}$ & $-0.0848^{* *}$ & $-0.0627^{* *}$ \\
& & $(0.0385)$ & $(0.0128)$ & $(0.0154)$ \\
\hline \hline
\end{tabular}

Note: $\quad$ Clustered standard errors in parentheses. ${ }^{*} p<0.10,{ }^{* *} p<0.05$.

EPR is multiplied by 100 and age is divided by 10 .

The key parameter of interest is $\delta$ capturing the autoregressive part of the employment process. For those with low and high education, the parameter is in the range 0.6-0.65, and for medium education it is below 0.5. This implies that deviations from the generic life-cycle path are only weakly persistent. For example for the medium educated, less than $5 \%$ of the initial impact remains after 6 years. This is suggestive that the endogenous persistence mechanism is not particularly strong within the young sample. Note that the autoregressive process does not fully capture the dynamic process, and therefore we report 
the impulse response functions below.

The estimation captures that men tend to have higher employment rates than women. The difference is declining in the educational level. Among highly educated, males and females have similar initial responses to business cycle shocks, but our estimates suggest that males recover faster as the coefficient $\beta_{3}$ on the interaction between gender and lagged output gap is negative. For medium and low educated, males typically experience a larger impact from shocks to output than females, but we also see larger lagged responses from output gaps suggesting that males also recover faster. Finally, young individuals are more vulnerable to the cyclical fluctuations in their early years on the labour market (the $\theta$ coefficient). This is also found for other countries (see e.g. Bell \& Blancflower (2011) for evidence for OECD countries).

From Figure 1 it is seen that the variability of employment is at about the same level for women and men. This suggests that the higher short-run sensitivity to GDP shocks of men is counterbalanced by their faster adjustment. However, the two sources of variation have very different implications for persistence. We consider this issue in more detail below.

\subsection{Results for the old sample}

The results for the old sample are reported in Table 2 . 
TABle 2: Results For the OlD SAMPle

\begin{tabular}{|c|c|c|c|c|}
\hline & & Low educ. & Medium educ. & High educ. \\
\hline \multirow[t]{2}{*}{$\delta$} & Lagged EPR $\left(e_{s, i, t-1}\right)$ & $0.958 * *$ & $1.036^{* *}$ & $0.745^{* *}$ \\
\hline & & $(0.0258)$ & $(0.0277)$ & $(0.0694)$ \\
\hline \multirow[t]{2}{*}{$\beta_{0}$} & Output gap $\left(y_{t}\right)$ & $0.319^{* *}$ & $0.313^{* *}$ & $0.195^{* *}$ \\
\hline & & $(0.0294)$ & $(0.0279)$ & $(0.0243)$ \\
\hline \multirow[t]{2}{*}{$\beta_{1}$} & Lagged output gap $\left(y_{t-1}\right)$ & $-0.0966^{* *}$ & $-0.137^{* *}$ & $-0.112^{* *}$ \\
\hline & & $(0.0327)$ & $(0.0311)$ & $(0.0173)$ \\
\hline \multirow[t]{2}{*}{$\beta_{2}$} & Output gap $\times$ male $\left(y_{t} \cdot s_{i}\right)$ & $0.234^{* *}$ & $0.220^{* *}$ & $0.0916^{* *}$ \\
\hline & & $(0.0312)$ & $(0.0265)$ & $(0.0403)$ \\
\hline \multirow[t]{2}{*}{$\beta_{3}$} & Lagged output gap $\times$ male $\left(y_{t-1} \cdot s_{i}\right)$ & $-0.395^{* *}$ & $-0.369^{* *}$ & -0.0409 \\
\hline & & $(0.0416)$ & $(0.0405)$ & $(0.0324)$ \\
\hline \multirow[t]{2}{*}{$\theta$} & Age dummy $(50-54) \times$ output gap & $0.0857^{* *}$ & $0.0696^{* *}$ & $0.0600^{* *}$ \\
\hline & & $(0.0274)$ & $(0.0323)$ & $(0.0204)$ \\
\hline \multirow[t]{2}{*}{$\theta$} & Age dummy $(55-57) \times$ output gap & $0.183^{* *}$ & $0.239^{* *}$ & $0.153^{* *}$ \\
\hline & & $(0.0477)$ & $(0.0391)$ & $(0.0357)$ \\
\hline \multirow[t]{2}{*}{$\kappa_{0}$} & Age $\left(a_{i, t}\right)$ & 0.160 & 0.507 & $12.14^{* *}$ \\
\hline & & $(2.402)$ & $(2.280)$ & $(1.968)$ \\
\hline \multirow[t]{2}{*}{$\kappa_{1}$} & Age squared $\left(a_{i, t}^{2}\right)$ & -0.151 & -0.130 & $-1.384^{* *}$ \\
\hline & & $(0.236)$ & $(0.228)$ & $(0.213)$ \\
\hline \multirow[t]{2}{*}{$\psi$} & Male $\left(s_{i}\right)$ & $0.538^{* *}$ & $0.158^{* *}$ & 0.0425 \\
\hline & & $(0.147)$ & $(0.0411)$ & $(0.0675)$ \\
\hline \multirow[t]{4}{*}{$\gamma$} & Transition scheme $\left(z_{i, t}\right)$ & $-0.423^{* *}$ & -0.138 & $-0.361^{* *}$ \\
\hline & & $(0.130)$ & $(0.140)$ & $(0.113)$ \\
\hline & Trend & -0.00758 & $-0.0353^{* *}$ & 0.00933 \\
\hline & & $(0.0155)$ & $(0.0136)$ & $(0.00716)$ \\
\hline
\end{tabular}

Note: Clustered standard errors in parentheses. ${ }^{*} p<0.10,{ }^{* *} p<0.05$

EPR is multiplied by 100 and age is divided by 10 .

The autoregressive parameter $\delta$ is much higher for the old sample for all three educational groups. In the case of low and medium education, the coefficient is not significantly different from one, suggesting a strong degree of persistence in the response of employment to business cycle shocks. For the highly educated the parameter is somewhat lower, and although larger than for the similar educational group in the young sample, the age difference is much smaller for this group. 
The initial employment effect of a business cycle shock is lower for the highly educated. The significant interaction between gender and the business cycle shock, especially for the low and medium educated, confirms that men are on impact more exposed to business cycle shocks than women, also for the old sample. This is also the case for the impact of last periods output gap. For highly educated there is very little difference in the adjustment process for males and females although males might be more affected on impact.

Finally, we see that the employment response to shocks to the economy is generally increasing with age within the old sample. Since employment levels are much more sensitive to cyclical fluctuations with age, it follows that variations around the mean increase with age, cf. Figure 2. ${ }^{16}$ This finding is robust across educational levels, but is stronger for the low and medium educated.

Relative to the younger sample it is not obvious who is most affected by recessions. The EPRs of young cohorts are more sensitive to the business cycle situation (especially for the very young), but they recover relatively fast. The EPRs of the older cohorts are highly persistent but less sensitive to the business cycle. The results suggest that young individuals are more exposed to unemployment risks than older individuals during recessions, but that the duration of the average unemployment spell is much longer for the elderly. This could be explained by the fact that non-employment becomes an absorbing state for some old individuals via e.g. early retirement (see e.g. Gruber \& Wise, 1999). These issues are explored further in relation to the impulse response functions reported below.

\section{Are the employment costs of recessions cohort spe- cific?}

The notion of lost generations can be interpreted in different ways. We consider two interpretations. The first is that cohorts exposed to a decline in employment at a particular age never fully recover from this set-back, and the employment rate will, as a consequence, be lower permanently. The other interpretation is that particular cohorts have been exposed to a sequence of (large) negative employment shocks, and although they may recover in terms of employment at a later stage, they still have experienced a

\footnotetext{
${ }^{16}$ Note that the high $\delta$ also generates increasing variability around the mean for later ages.
} 
significant decline in life-time employment. We consider these two notions of lost generations in turn. Finally, we point out that by virtue of the overlapping generations the effect of persistency on the aggregate employment rate is mitigated by the fact that particular generations retire from the labour market.

\subsection{Endogenous persistence}

To explore endogenous persistence we consider impulse response functions for employment rates. The impulse response function is generated by exposing employment to a temporary exogenous business cycle shock ${ }^{17}$ (a one-time change in the output gap) and evaluating the employment path implied by the dynamics inherent in (1). In this sense the response is derived from endogenous mechanisms separate from the properties of the shock (which in itself only justifies a one-time change in employment and subsequent adjustment). The figures below plot the deviations from the underlying counter-factual process where there is no shock, such that the figures show how the employment rate deviates from its standard life-cycle path as a consequence of the temporary shock.

\footnotetext{
${ }^{17}$ The shock is a $1 \%$ output gap in the impact year. In subsequent years the output gap is set to zero.
} 
Figure 5: Impulse Response functions for AGes 20- And 45-
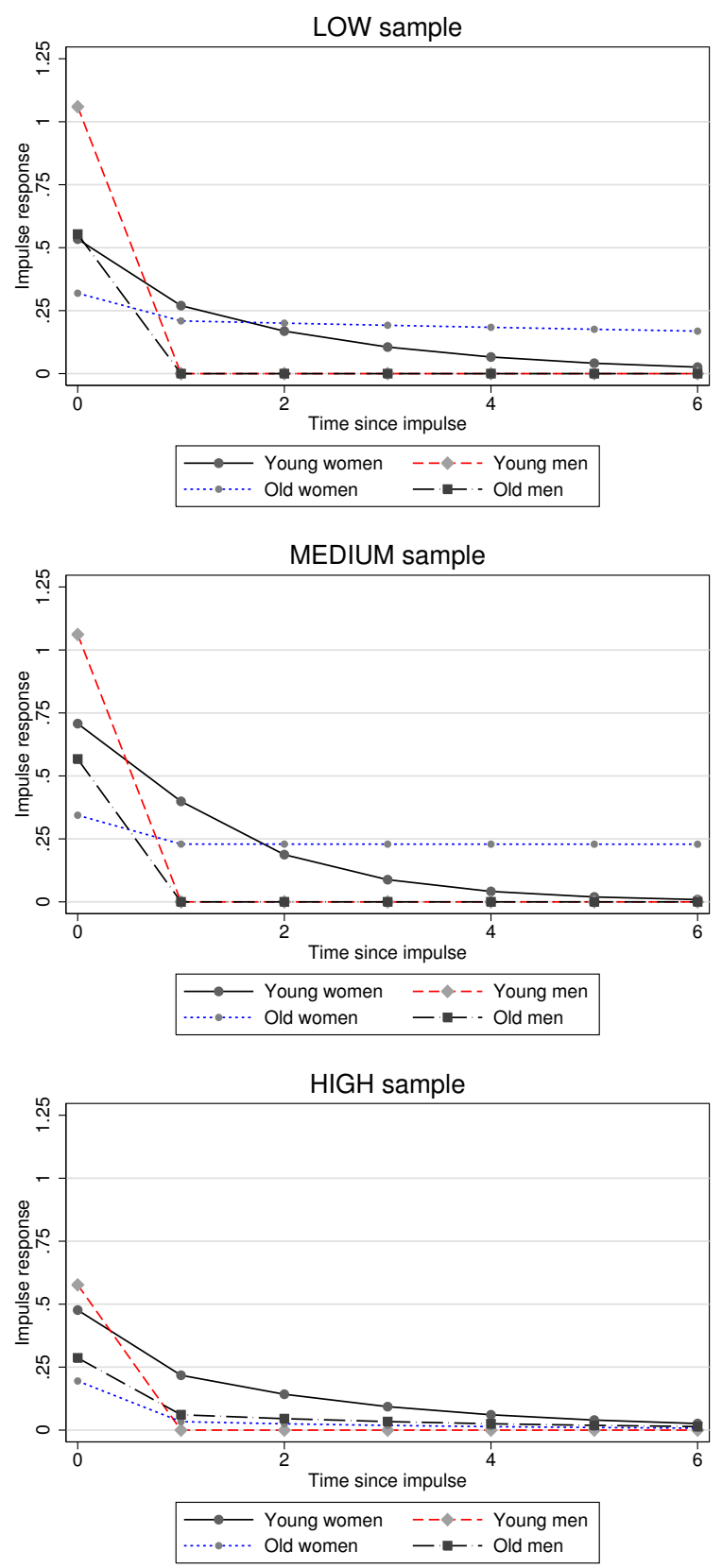

Note: The impulse responses for the young have impact at the age of 20 , and for the old at the age of 45 . The panels only include impulse responses significantly different from zero (10\% level). Standard errors are found by bootstrapping.

The impulse response functions show a clear ordering by education where individuals with low or medium education allevels generally are more sensitive to output fluctuations; this is especially so for males. In general, young males are more responsive for all levels 
of education in the short run compared to other groupings. The initial response for males is similar for low and medium educated but smaller for the highly educated.

Our estimates interestingly suggest that already in the year following the output shock the EPR for young males has fully recovered irrespective of educational level. Older males with low or medium education also recover fast and their initial response is much lower (recall that this is at the age 45 , the prime aged workers).

For women the recovery process is slower except for the highly educated older aged women. Six years after the impulse only $6 \%$ of the initial shock remains for young women whereas for the older women $50 \%$ of the initial shock still remains for the low educated. In fact our estimates suggest that older females with low or medium education never recover fully. Young females have a slower adjustment process, but eventually they catch up completely in all samples.

It is important to note that the employment response for the cohort depends critically on the age at which the shock occurs. Above we have the shock to appear at the earliest age within the two age groups (20 and 45, respectively). In Table 3 accumulated employment changes for different impact ages are reported. This is at the same time an alternative way by which to summarize the effects of the endogenous persistence represented in the impulse responses above. Table 3 shows the accumulated deviations from mean employment rates 6 years after a positive shock to an output gap of $1 \%$.

Table 3: Accumulated effect on employment rates (In Per Cent) 6 years AFter A POSITIVE BUSINESS CYCLE SHOCK OF $1 \%$

\begin{tabular}{ccccccc}
\hline \hline & \multicolumn{2}{c}{ Low education } & \multicolumn{2}{c}{ Medium education } & \multicolumn{2}{c}{ High education } \\
Age at impact & Women & Men & Women & Men & Women & Men \\
\hline 20 & 1.21 & 0.94 & 1.45 & 0.96 & 1.06 & 0.78 \\
31 & 0.51 & 0.24 & 0.84 & 0.34 & 0.87 & 0.60 \\
45 & 1.45 & 0.76 & 1.54 & 0.84 & 0.30 & 0.48 \\
51 & 1.97 & 1.29 & 2.09 & 1.39 & 0.51 & 0.69 \\
\hline \hline
\end{tabular}

For all educational groups there is a U-pattern in age; that is, the accumulated deviations are largest if the shock appears for the youngest and the oldest. This brings out that the very young and old tend to carry the largest employment consequences of business cycle fluctuations. This finding complements the findings from Figure 4 by underlining that among the young it is the youngest who are most affected by business cycle shocks. 
The general insight from Table 3 is that business cycle shocks have important impacts on subsequent labour market performance in the short and medium term, and that this is particularly visible for low and medium educated, for older workers and for women. Consistent with the empirical micro economic literature we find that being exposed to a negative or positive shock to employment may have longer lasting effects on the future labour market status (see e.g. Doiron \& Gørgens (2008), Hartman et al. (2010), and Lesner (2013)).

\subsection{Generational effects of economic shocks}

Although endogenous persistence is not that strong for most groups it may still be that certain cohorts have been lost or are losing generations as a result of having been exposed to a sequence of adverse shocks during youth. Even though employment rates recover for the cohort, the life-time income may still be affected.

To quantify the effects of being exposed to business cycle shocks, realized employment rates are compared to employment rates in the counterfactual case with no output gaps. Figure 6 shows the accumulated employment deviations at ages 25, 30 and 35 for both men and women for low educated across birth years in the young sample. ${ }^{18}$ A generation with negative deviations at all three age levels has been affected particularly hard by unfavourable business cycle situations.

\footnotetext{
${ }^{18}$ We only show the results for low educated as these are generally most affected by business cycle shocks. The results for the other educational groups are of course available from the authors upon request.
} 

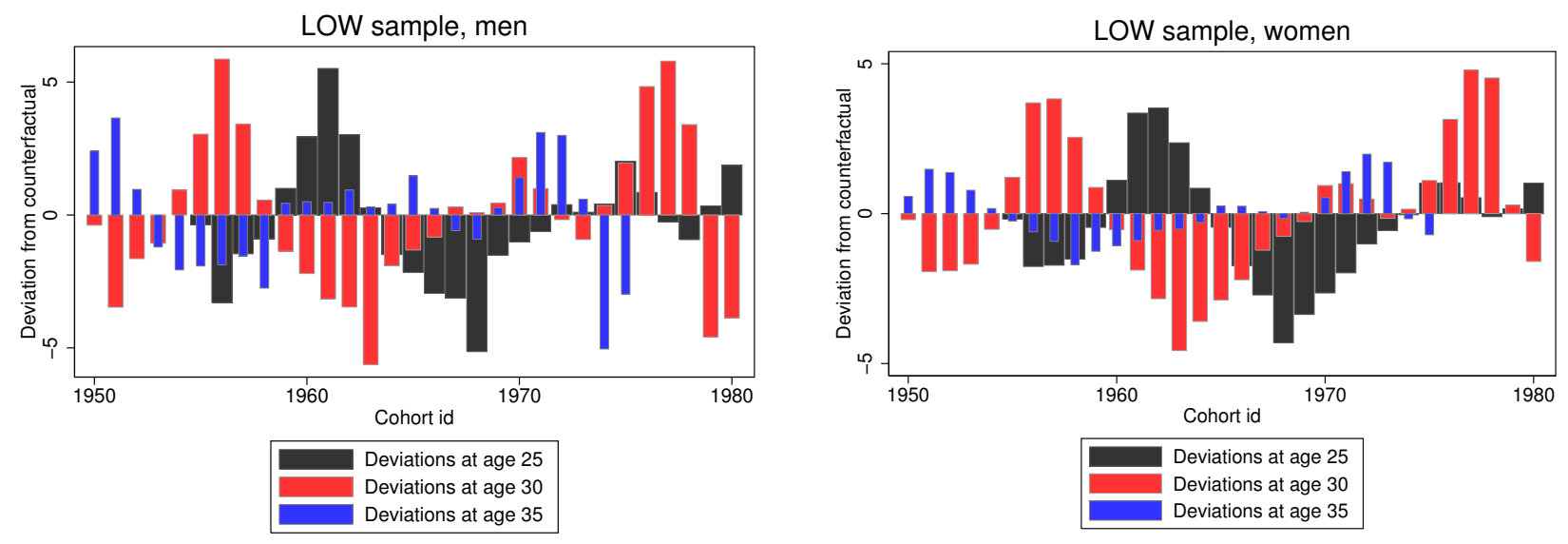

Note: Computed as deviations from the predicted EPR from the counterfactual EPR where no shocks occur. For each cohort we use the actual series of gaps in output which this particular cohort faced in their life-cycle and compare this to a situation with an output gap of 0 .

The lowest employment rate during the observation period was observed in 1993. This is clearly reflected in Figure 6. For, e.g., the cohort from 1963 there is a large negative deviation at age 30. A similar pattern is seen for the other cohorts. It is also clear for, e.g., the 1963 cohort that although they have a much lower employment rate at the age of 30 due to the economic slowdown in the early 1990s, they are back on track at age 35, where they actually have a slightly higher employment rate than the counterfactual situation.

Comparing across cohorts in Figure 6 it is not possible to identify a cohort which is consistently below or above the counterfactual employment level at all three age thresholds considered. ${ }^{19}$ In this sense it is not possible to identify a cohort that has carried a disproportionately large burden (gain) of business cycle fluctuations.

\subsection{Implication for macro level persistence}

The aggregate employment rate at a given point in time is a weighted average of the employment rates for the particular cohorts that are of working age at that point. Each year a new cohort enters and an old retires from the labour market. This implies that there is an important distinction to be made between persistence embedded in the

\footnotetext{
${ }^{19}$ This observation is not dependent on the specific ages reported in the figure. The pattern is consistent across different choices of ages.
} 
employment trajectory of a particular cohort, and the persistence arising at the aggregate level. Even if there is strong persistence at the cohort level, it will be less strong at the aggregate level where account is take of the fact that cohorts retire. This also points out that one should be careful in interpreting measures of persistence derived from aggregate employment measures. An explicit account of overlapping generations/cohorts may thus give a more detailed and precise assessment of persistence in the labour market than the standard time series approach suppressing this source of dynamics. This is also an argument in favour of the meso approach pursued in this paper.

To illustrate this Figure 7 displays persistence within the old sample. Consider a temporary shock to output which, in turn, affects employment for all cohorts within the sample in the given year. In the next year a new cohort has entered the old sample and one cohort has left, and so on and so forth. The results presented earlier in the article show that persistence is lower for younger cohorts and as approximation we assume there is no endogenous persistence for entering cohorts. Eventually, cohorts having been exposed to the shock are no longer present in the sample. The red bars in Figure 7 display the impulse response function for the employment for all in the old age group when account is taken of the overlapping generations dynamics. The black bar is the case where no account is taken of this dynamics. The figure illustrates why analysing the EPR over time, for instance for older workers, tends to misrepresent persistence.

Figure 7: Average Deviations from counterfactual EPR

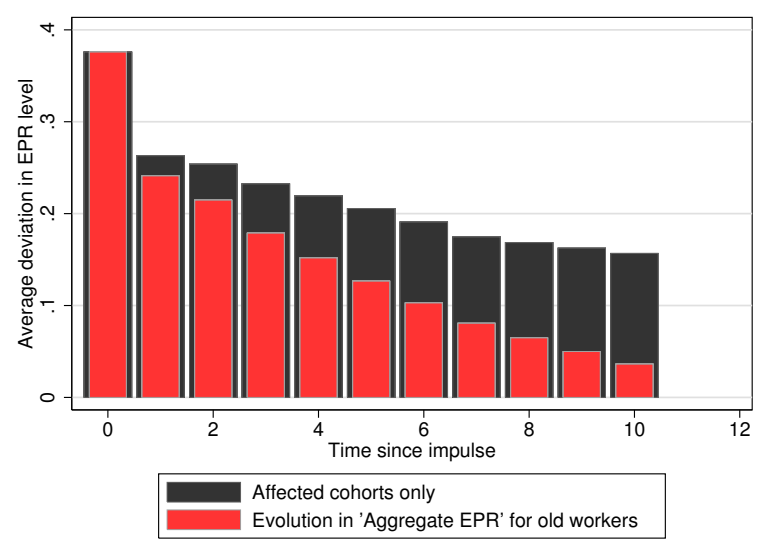

Note: The black bars follow the deviations from the predicted EPR from the counterfactual EPR where no shocks occur for affected cohorts only. The red bar follows the evolution of aggregate EPR in the old sample where a new unaffected cohort enters each time period and an affected one exits. 


\section{Concluding remarks}

The economic crisis and the steep increase in youth unemployment have received considerable attention. A particular concern is that the costs are embedded in particular cohorts entering the labour market during the slump, and that they therefore are becoming lost generations.

In discussing the employment consequences of slumps it is important to make a distinction between the impact effect and the adjustment process. We develop an empirical set-up which allows an explicit separation between the two and explicitly takes a cohort perspective building on the life-cycle pattern of labour market participation. The empirical analysis is performed on Danish data.

In line with the literature we find that employment of both young and old are particularly sensitive to the business cycle situation. For low and medium educated male individuals a $1 \%$ drop in the output gap implies a $1 \%$ drop in the level of employment ratio in the year of the shock. The effects are the more pronounced for less educated. However, there are important differences in the adjustment process across the groups which have crucial implications for the discussion of lost generations. In general our estimates suggest that the adjustment process is relatively quick. Male employment rates recover almost immediately, whereas $20 \%$ of the initial smaller drop in women employment rates remain 3 years after the shock. Although men experience larger initial impact, our estimates suggest that the young women suffer more in terms of accumulated losses due to the slower adjustment process.

The notion of lost generations can be interpreted in two ways. One interpretation is that a temporary (large) employment drop will imply that employment for the cohort is affected for a sequence of years, possibly permanently. This is so-called endogenous persistence where various mechanisms in the labour market imply that a cohort never or only very slowly recovers from set-backs to employment. Another interpretation is that particular cohorts have been exposed to sequences of adverse shocks which over a longer period have implied below normal employment rates, and although employment rates later recover these cohorts have experienced significant losses in life-time income. We do not find strong support for lost generations in either interpretation. Endogenous persistence is in general weak, with the exception of older women with low or medium levels of education. While some cohorts have been exposed to negative shocks over a number of years it tends to even out in the sense that they are later exposed to favourable 
shocks.

There are two important caveats to our findings in relation to the general discussion about the financial crisis and youth unemployment. The present recession is both deeper and more prolonged compared to the business cycle downturns in our sample. Therefore the potential loss of human capital and the scarring effect due to employment losses may be both stronger and of a different nature than previous crises in our sample. Another important point is that the Danish labour market is rather flexible in a European context. Gross job creation and destruction rates are high in a comparative perspective, and have remained so during the crisis. It may thus be easier for youth to enter the labour market when there always is a relatively high level of job openings. Accordingly the dynamics in the Danish labour market may differ in important respects from other countries, especially in relation to youth. It is an interesting question for future research to explore more carefully how dynamics and persistence mechanisms depend on labour market institutions and policies. 


\section{$7 \quad$ References}

Andersen, T.M., 2004, Real and Nominal Propagation of Nominal Shocks, Economic Journal, 114, 174-195.

Anderson, T.W. and C. Hsiao, 1981, Estimation of Dynamic Models with Error Components, Journal of the American Statistical Association, 589-606.

Arrelano, M. and Bond, S., 1991, Some Tests of Specification for Panel Data: Monte Carlo Evidence and an Application to Employment Equation, Review of Economic Studies, 58, 277-297.

Arellano, M. and Honore, B., 2001, Panel Data Models: Some Recent Developments, Handbook of Econometrics vol. 5

Arellano, M. and O. Bover, 1995, Another Look at the Instrumental Variable Estimation of Error-Components models, Journal of Econometrics, 68, 29-51.

Bell, D. and D. Blanchflower, 2011, Young People and the Great Recession, Oxford Review of Economic Policy, 27.

Bernal-Verdugo, L.E., D. Furceri, and D. Guillaume, 2012, Crisis, Labour Market Policy, and Unemployment, IMF Working Paper 12/65.

Blanchard, O., 2006, European Unemployment: The Evolution of Facts and Ideas, Economic Policy, 21, 5-59.

Blundell, R. and S. Bond, 1998, Initial Conditions and Moment Restrictions in Dynamic Panel Data Models, Journal of Econometrics, 87, 115-143.

Blundell, R. and S. Bond., 2000, GMM Estimation with Persistent Panel Data: An Application to Production Functions, Econometric Reviews, 19, 321-340.

Cogley, T. and J.M Nason, 1995, Output Dynamics in Real-Business-Cycle Models, American Economic Review, 85, 492-511.

Doiron, D. and T. Gørgens, 2008, State Dependence in Youth labour Market Experiences, and the Evaluation of Policy Interventions, Journal of Econometrics, 145, 81-97.

Duval, R., J. Elmeskov, and L. Vogel, 2006, Structural Policies and Economic Resilience to Shocks, OECD Working Paper no $56 \%$.

Genda, Y., A. Kondo and S. Ohta, 2008, Long-Term Effects of a Recession at labour Market Entry in Japan and the United States, Journal of Human Resources, 45, 157-196.

Giuliano, P., and T. von Wachter, 2012, Does a Persistently Higher Unemployment Rate Make a Difference? Wage Growth and Job Mobility in Germany, France, and the United States, forthcoming in Journal of Economic Perspectives. 
Gruber, J. and D. Wise, 1999, Social Security around the World, NBER conference report.

Guichard, S. and E. Rusticelli, 2010, Assessing the Impact of the Financial Crisis on Structural Unemployment in OECD Countries, OECD Economics Department Working Paper, No. 767, OECD Publishing, Paris.

Hairault, J.-O., F. Langot, and T. Sopraseuth, 2010, Distance to Retirement and Older Workers' Employment: The Case for Delaying the Retirement Age, Journal of the European Economic Association, 8(5), 1034-1076.

Hartman, L., L. Liljeberg, and O. Skans, 2010, Stepping-Stones, Dead-Ends, or Both? An Analysis of Swedish Replacement Contracts, Empirical Economics, 38, 645-668.

Hayakawa, K., 2006, Small Sample Bias Properties of the System GMM Estimator in Dynamic Panel Data Models, Economic Letters, 95, 32-38.

Hayashi, F., 2000, Econometrics, Princeton University Press.

Heckman, J. J. and G. Borjas, 1980, Does Unemployment Cause Future Unemployment? Definitions, Questions and Answers from a Continuous Time Model of Heterogeneity and State Dependence, Economica, 47, 247-283.

IMF, 2012, World Economic Outlook.

Jorda, O., 2005, Estimation and Inference of Impulse Responses by Local Projections, American Economic Review, 95(1), 161-182.

Lesner, R. W., 2013, Does Labour Market History Matter?, manuscript, Aarhus University.

Liu, K., K.G. Salvanes and E.Ø. Sørensen, 2013, Bad Times at a Tender Age - How Education Dampens the Impact of Graduating in a Recession, Nordic Economic Policy Review (to appear).

Lockwood, B., 1991, Information Externalities in the Labour Market and the Duration of Unemployment, Review of Economic Studies, 58, 733-753.

OECD, 2012, OECD Economic Outlook.

Oreopoulos, P., T. van Watcher, and A. Heisz, 2012, The Short- and Long-Term Career Effects of Graduating in a Recession, American Economic Journal: Applied Economics, $4(1), 1-29$.

Pissarides, C. A., 1992, Loss of Skill During Unemployment and the Persistence of Employment Shocks, Quarterly Journal of Economics, 107, 1371-1391.

Roed, K., 1997, Hysteresis in Unemployment, Journal of Economic Surveys, 11, 389418. 
Roodman, D., 2009, A Note on the Theme of Too Many Instruments, Oxford Bulletin of Economics and Statistics, 71, 135-158.

Roodman, D, 2009: How to do xtabond2: An Introduction to Difference and System GMM in Stata, Stata Journal, 9,1.

Teulings, C. and N. Zubanov, 2010, Is Economic Recovery a Myth? Robust Estimation of Impulse Responses, CESifo Working Paper Series $302 \%$. 


\section{Appendix A}

Figure A.1: Output gap Series for the SAMple Period

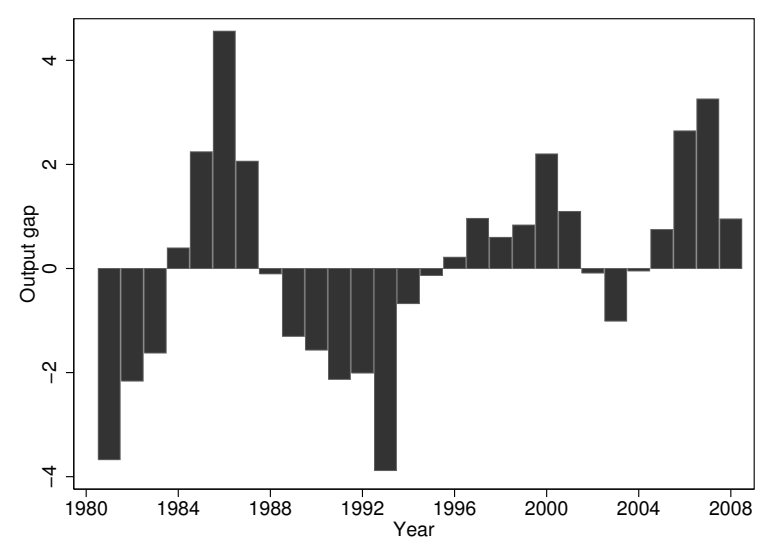

\section{Data}

The empirical analysis is based on IDA, a Danish register-based annual matched employer-employee panel covering the whole labour force in the years 1980-2008. The dataset is kept by Statistics Denmark. The data are confidential, but access is not exclusive. The unit of observation is a given individual in a given year with measurements generally referring to the last week of November. From this data set we identify the cohortrelationship of each individual and we determine the educational level of the individual based on his obtained education around age 30 or higher if not present. As explained in the text above, we proceed by constructing 3 samples based on the educational level of the individual (low, medium and high). ${ }^{20}$

Table A.1: SAmple Size

\begin{tabular}{cccc}
\hline \hline Men & Low educ. & Medium educ. & High educ. \\
\hline Young sample & $7932(22 \%)$ & $15229(43 \%)$ & $12590(35 \%)$ \\
Old sample & $10792(33 \%)$ & $14542(44 \%)$ & $7633(23 \%)$ \\
\hline & & & \\
Women & Low educ. & Medium educ. & High educ. \\
\hline Young sample & $6638(19 \%)$ & $11805(34 \%)$ & $15984(46 \%)$ \\
Old sample & $13329(41 \%)$ & $11357(35 \%)$ & $7948(24 \%)$ \\
\hline \hline
\end{tabular}

\footnotetext{
${ }^{20}$ For a more detailed documentation on IDA see http://www.dst.dk/HomeUK/Guide/documentation/Varedeklaratione emnegruppe/emne.aspx?sysrid=1013.
} 
In each sample we calculate the employment to population ratio (EPR) for each cohort in a given year separately for males and females. We use the cohorts with individuals born from 1935 to 1980 (this secures a minimum of 9 observations per cohort). Note that our sample has truncated spells, i.e. young generations with incomplete spells and older generations where the previous employment spell is not known (see Figure A.1).

The definition of employment relates to the status of the individual in the labour market (i.e. does the person have a job), and furthermore we have an earnings requirement (properly deflated to the given year) such that our employment variable captures something as close as possible to regular employment over the past year. 
Figure A.1: Presentation of Cohorts in the sample

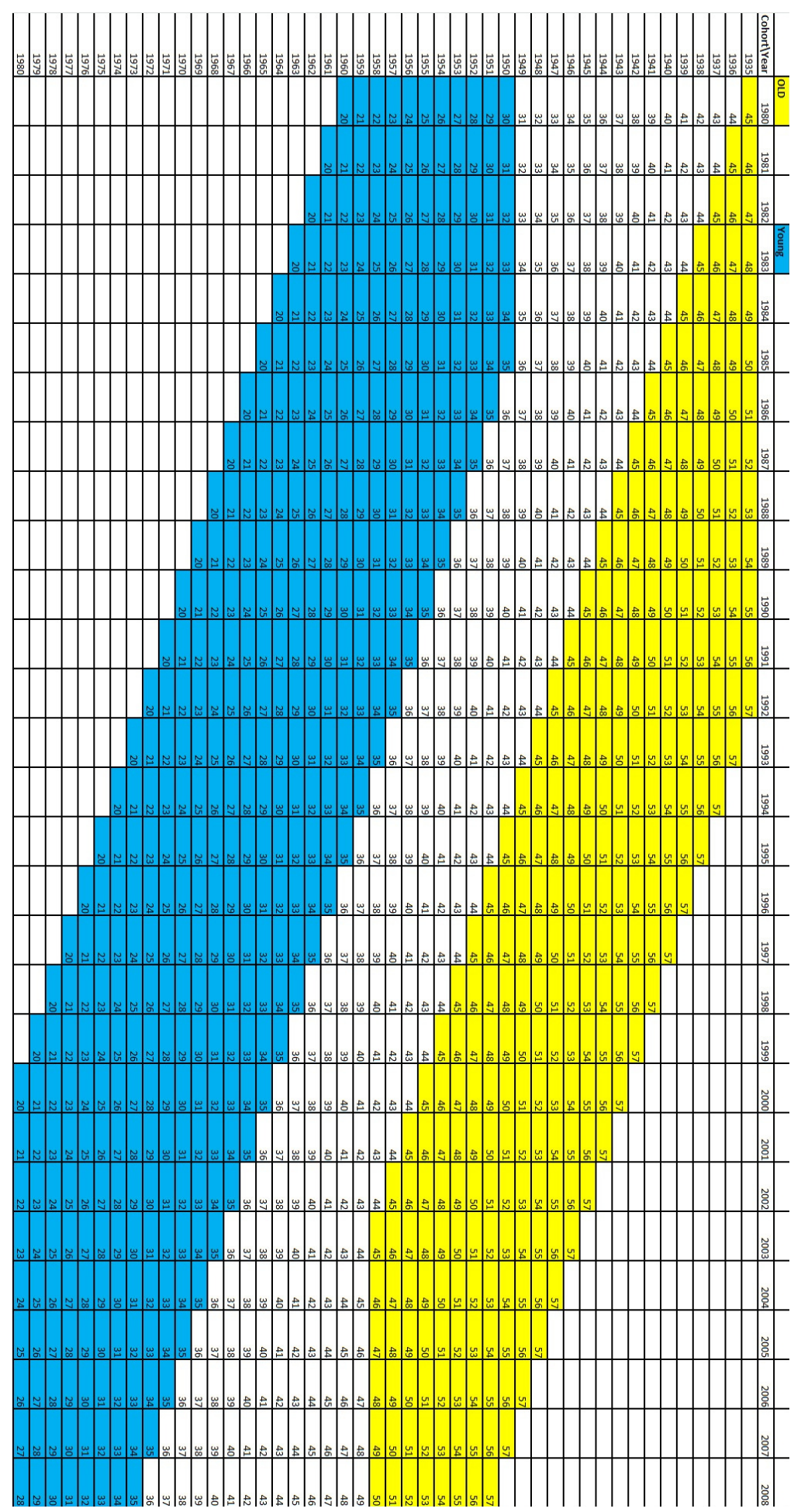




\section{Appendix B: Robustness analysis}

Table B.1 reports the value of the Hansen test statistic and the number of observations and "instruments" (moment conditions) used in each sample for our preferred model. Generally we do not reject the identifying assumptions of our model for the overidentifying restrictions. Due to the relatively long sample period (1980-2008) in our data the instrument count becomes large in our preferred model, and this limits the validity of the conventional tests. As already mentioned we have tried to estimate specifications where we limit the number of instruments dramatically in order to analyse the performance of our model in these settings. Table B.2 reports the test results from a specification that uses fewer moment conditions but generates very similar estimates to those reported in the text above. We have also tried specifications that rely on later lags in the instrument set to allow for presence of higher order serial correlation (which the Arrelano Bond test (AB test) indicates for some specifications below). This does not change the main predictions of our model, but it is important to keep in mind that restricting the instrument count increases the variability of our estimates.

Table B.1: Preferred models

\begin{tabular}{|c|c|c|c|c|c|c|}
\hline & \multicolumn{3}{|c|}{ Toung sample } & \multicolumn{3}{|c|}{ Old sample } \\
\hline & Low ed. & Medium ed. & High ed. & Low ed. & Medium ed. & High ed \\
\hline Hansen Test & 40.48 & 39.39 & 55.95 & 31.08 & 40.72 & 34.21 \\
\hline $\mathrm{AB}$ test $\mathrm{AR}(2)$ & $-1,23$ & $-1,13$ & $-3,88$ & 0,03 & $-1,26$ & -0.07 \\
\hline Difference Hansen ${ }^{21}$ & 0.82 & -9.98 & 3.15 & 2.14 & 6.87 & -2.57 \\
\hline Number of observations & 764 & 764 & 534 & 520 & 520 & 520 \\
\hline Number of "instruments" & 118 & 116 & 85 & 107 & 107 & 107 \\
\hline
\end{tabular}

\footnotetext{
${ }^{21}$ The difference between the two Hansen tests when we exclude the levels equation and when we include it in the estimation.
} 
Table B.2: When Number of instruments ARe minimized

\begin{tabular}{|c|c|c|c|c|c|c|}
\hline & \multicolumn{3}{|c|}{ "Young sample } & \multicolumn{3}{|c|}{ Old sample } \\
\hline & Low ed. & Medium ed. & High ed. & Low ed. & Medium ed. & High ed. \\
\hline Hansen Test & 55.15 & 56.24 & 57.92 & 41.70 & 42.25 & 38.96 \\
\hline $\mathrm{AB}$ test $\mathrm{AR}(2)$ & -0.74 & -0.20 & -2.84 & 1.22 & 0.13 & 0.19 \\
\hline Difference Hansen ${ }^{22}$ & -1.23 & -4.01 & -0.71 & -0.85 & -1.00 & 1.12 \\
\hline Number of observations & 764 & 764 & 534 & 520 & 520 & 520 \\
\hline Number of "instruments" & 71 & 71 & 60 & 68 & 68 & 68 \\
\hline
\end{tabular}

We have explored various other dynamic specifications of our model in order to determine whether our results are driven by badly specified dynamics. Generally we find this not to be the case. Our estimation approach allows us to distinguish between immediate and adjustment costs from recessions.

Below we will present the adjustment process after a shock to output implied by our estimated model. We do this by using impulse responses functions $(\operatorname{IRF}(k)$ where $k$ denotes periods since impulse). Naturally the IRFs rely heavily on the correct specification of the underlying model and the imposed linearity. Therefore, as a further robustness check, we follow the local projections approach proposed by Jorda (2005) and extended by Teulings and Zubanov (2010). The method is a good alternative to calculating IRFs analytically from an estimated dynamic model as it relies less on a particular specification of a dynamic model. The price is that it is no longer possible to separate the different costs from recessions. The local projections method estimates $\operatorname{IRF}(\mathrm{k})$ directly from the data for each $\mathrm{k}$ instead of using the same estimated analytical model. Essentially the local estimate of the $\operatorname{IRF}(\mathrm{k})$ of the cohort EPR is the coefficient on output gap at time $\mathrm{t}$ in a regression on $E P R_{t+k}$. We did not find substantial differences in impulse response patterns that would change the overall conclusions of our analysis from above, although there are some local differences (figures are available upon request).

\section{Sample selection and initial conditions}

As a robustness test we have reestimated our model excluding the oldest of our cohorts to test whether our results are simply due to a selected sample ${ }^{23}$. We still find very similar

\footnotetext{
${ }^{22}$ The difference between the two Hansen tests when we exclude the levels equation and when we include it in the estimation.

${ }^{23}$ We have also tried restricting the sample in the year domain by deleting observations from the 80's or 00's. Same conclusion applies.
} 
estimates of $\delta$ and thereby a long adjustment process for older workers from shocks to output.

As already mentioned, the empirical literature suggests that level of education is likely to be affected by the state of the economy. One standard argument is that the marginal costs of further education are likely to be lower in a recession due to lower employment chances. This implies that the sample selection rule may be endogenously determined, and this could affect our results if it implies that some cohorts will respond differently to variations in output than they would had the economic environment been different in their youth. ${ }^{24}$ To get an idea about the magnitude of such entry effects, we have made simple regressions where we regress the ratio of highly educated individuals in a cohort on the values of our business cycle measure around ages 20-25. We find a clear trend in our results (in the sense that younger cohorts obtain more education), and we find a small positive and marginally significant effect from our BC measure on the ratio of individuals with low education. Therefore, as a further robustness check, we have also estimated our model on data where we do not stratify on educational levels. The estimates and the implied impulse responses are in the same order of magnitude as those we find for low and medium educated. Figure B.1 shows the implied impulse responses that we obtain from this estimation exercise. We therefore conclude that our sample selection on educational level have not biased our conclusions.

Finally our estimation procedure crucially relies on correctly specified moment conditions which could be interpreted as a restriction on the initial conditions process. Intuitively speaking, if the cohort starts out bad and this affects the rest of its lifecycle such that responses to the business cycle change, our estimates are likely to be biased.

\footnotetext{
${ }^{24}$ Obviously the time-invariant part of this effect is absorbed in the unobserved heterogeneity term, but time-varying effects could bias our results.
} 
Figure B.1: Impulse Responses in RAW DATA Before EduCATion

STRATIFICATION

ONE sample

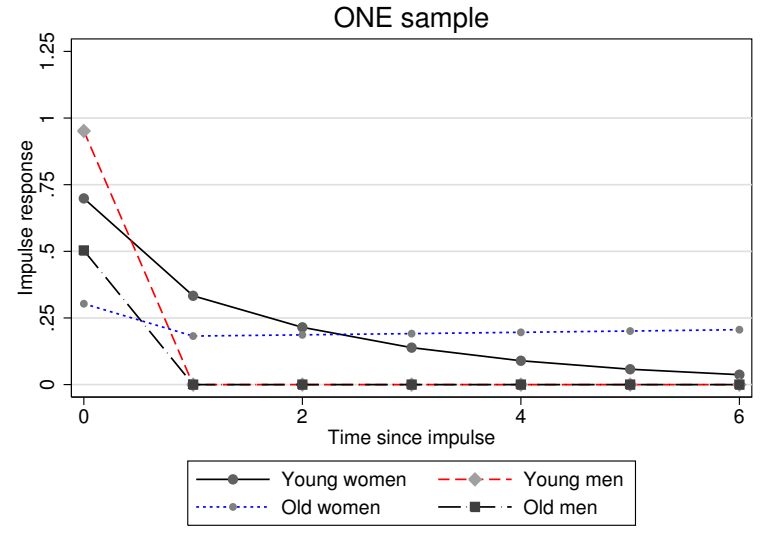

Note: The impulse responses for the young have impact at the age of 20 , and for the old at the age of 45 . The panels only include impulse responses significantly different from zero (10\% level). Standard errors are found by bootstrapping. 